\title{
Techniques of UDT
}

UDT consists of following measurements:

Cystometry is a measurement of pressure in the bladder during bladder filling and is done by introducing a tube into the bladder which is connected to an external pressure measuring device. While the bladder is filled it demonstrates NDO, measures the extensibility of the bladder wall, the pressure point at leakage and can be used to help evaluate filling sensation. When limited resources do not allow purchasing an electronic equipment, a cheap one channel cystometry device permits to gain valuable information. But interpretation is easier when bladder pressure measurement is combined with measurement of intrarectal/intraabdominal pressure as discussed below.

Uroflowmetry measures the flow rate during voiding. It needs voluntary control of voiding which may not be the case in SCI individuals.

Pressure-flow study measures detrusor pressure during voiding. It can assess several functional LUT parameters in patients with other neuropathy but is less easy to perform in many SCI patients who cannot void on command.

Imaging-urodynamic or video-urodynamic study under X-rays or with ultrasonography has the great advantage to provide combined functional and anatomical data. Important findings are summarised in the International SCI Basic Urodynamic data set.

Ambulatory UDT is a newer way to follow LUT function during daily activities in the wheelchair, but needs more specialised equipment.

Urethral pressure profile measures urethral pressure over the length of the urethra. It is rarely used in SCI individuals.

It is strongly advised to adapt the UDT technique to the neurologic condition after SCI. 